\title{
72. NEÓFITOS NUEVOS O INTERESANTES PARA LA FLORA ALICANTINA
}

\author{
Elena CAMUÑAS y Manuel B. CRESPO
}

Neophytes new or rare for the flora of Alicante ( $E$ of Spain).

Palabras clave. Neófitos, corología, flora, Alicante, España.

Key words. Neophytes, chorology, flora, Alicante, Spain.

Como resultado de las recolecciones que se vienen efectuando en la comarca de l'Alacantí (Alicante) se ha constatado la presencia de diversos neófitos que suponen novedades o citas de interés para la flora alicantina. En todos los casos parece interesante realizar un seguimiento de estas poblaciones para constatar su grado de naturalización en el futuro.

Los diferentes taxones se presentan en orden alfabético. Las plantas que constituyen novedad provincial se indican con un asterisco delante del nombre de la provincia. Para la nomenclatura y autoría de los mismos se acepta lo indicado en Flora Iberica (Castroviejo et al., 1986-1998) y para los taxones no recogidos en esta obra se sigue fundamentalmente a Mateo \& Crespo (1998). Las abreviaturas de los autores se presentan según Brummitt \& Powell (1992).

Los pliegos testigo de los taxones citados están depositados en el herbario $\mathrm{ABH}$ de la Universidad de Alicante.

\section{Bidens aurea (Aiton) Sherff}

ALICANTE: Alicante, La Goteta [30S YH24], 26-IV-1979, A. Rigual (ABH 21027). Ampliación de área.

Planta originaria de Centroamérica, que hasta ahora sólo se conocía en Alicante de las comarcas más septentrionales (Calduch, 1973; Mateo \& Crespo, 1988; Alonso, 1996). Con esta nueva localidad se amplía notablemente su distribución hacia los territorios semiáridos meridionales.

\section{Bidens pilosa $\mathrm{L}$.}

ALICANTE: Alicante, ctra. Hogar Provincial, 30SYH2252, 40 m, 5-IX-1996, E. Camuñas \& M.B. Crespo (ABH 19758). Ampliación de área.

Planta de origen neotropical, que hasta ahora sólo se conocía en Alicante de las comarcas septentrionales más húmedas y litorales (Nebot et al., 1990; Pérez Badia et al., 1994). La nueva localidad constituye una importante ampliación de su distribución hacia los territorios semiáridos meridionales, donde forma parte de herbazales higro-nitrófilos estivales correspondientes a la alianza Chenopodion rubri Tüxen ex Poli \& Tüxen 1960 corr. Kopecky 1969.

\section{"Bidens tripartita L."}

Este taxón fue indicado para la flora de la provincia de Alicante por Rigual (1984). Sin embargo, tras el estudio del material que dio origen a dicha referencia se ha podido constatar que corresponde realmente a Bidens aurea (Aiton) Sherff, por lo que, según los datos existentes, habrá de considerarla como ausente del catálogo provincial alicantino. En este sentido Mateo \& Crespo (1998) ya indican como dudosa su presencia en la Comunidad Valenciana y Bolòs \& Vigo (1996) no la consideran presente en dicho territorio.

\section{Cannabis sativa $\mathrm{L}$.}

ALICANTE: Alicante, Castillo de Santa Bárbara, 30SYH2047, 90 m, 21-XII-1996, E. Camuñas \& M.B. Crespo (ABH 32225). Alicante,

Trabajo financiado a cargo del convenio de investigación ALICANT1.97T suscrito entre el Excmo. Ayuntamiento de Alicante y la Universidad de Alicante, y del proyecto GV-C-RN-12-069-96 de la Generalitat Valenciana. 
Playa de San Juan, 30SYH25, 20-VIII-1989, cultivada, A. Rigual (ABH 20484). $2^{\text {a }}$ cita provincial.

Planta de origen asiático, que hasta ahora sólo se conocía de las comarcas alicantinas meridionales, a partir de ejemplares cultivados mencionados por Rigual (1972). Sin embargo, en la actualidad se encuentran con cierta frecuencia plantas asilvestradas, probablemente a partir de semillas utilizadas en la alimentación de pequeñas aves. En las localidades aquí aportadas participa en comunidades de Hordeion leporini $\mathrm{Br}$.- $\mathrm{Bl}$. in $\mathrm{Br}$.Bl et al. 1936, donde no parece llegar a naturalizarse. Dado lo escaso y puntual de los ejemplares encontrados, deberá considerarse de momento como planta adventicia.

\section{Cenchrus ciliaris L.}

ALICANTE: Alicante, Babel, prox. Gasolinera ctra. Elche, 30SYH1846, 5 m, 13-XI-1997, E. Camuñas et al. (ABH 38552). San Vicente del Raspeig, ctra. a Villafranqueza, 30SYH1752, $90 \mathrm{~m}$, 22-XI-1997, M.B. Crespo \& E. Camuñas (ABH 38556). $2^{\text {a }}$ cita provincial.

Originaria del noroeste africano, su primera referencia alicantina corresponde a los ejemplares introducidos para fijar los taludes del autovía A-7, a su paso por los municipios de El Campello-San Juan (Pérez Badia et al., 1994). En las nuevas localidades aportadas, esta planta se presenta de modo espontáneo, creciendo en bordes de camino y terrenos baldíos, en ambos casos en comunidades de Bromo-Oryzopsion miliaceae O. Bolòs 1970. Parece que comienza una lenta expansión en los territorios peninsulares más cálidos.

Cortaderia selloana (Schult. \& Schult. f.) Asch. \& Graebn.

ALICANTE: Alicante, Barranco de las Ovejas, 30SYH1550, 70 m, 1-X-1996, M.B. Crespo \& E. Camuñas (ABH 30050). Alicante, prox. C.I.R. Rabasa, 30S YH1750, 90 m, 7-VIII-1998, E. Camuñas \& M.B. Crespo (ABH 39633). Ampliación de área.

Especie de origen sudamericano ampliamente utilizada en jardinería, que ocasionalmente puede asilvestrarse en las proximidades de los núcleos urbanos. En las dos localidades indicadas se instala en zonas con notable humedad edáfica, aunque siempre a través de ejemplares aislados. El hecho de que en ocasiones se escape de cultivo no parece suponer un problema en las comarcas centrales de Alicante, dados los requerimientos hídricos de la especie y las condiciones semiáridas de dicho territorio, desfavorables para la expansión de esta planta. Un comportamiento similar fue indicado por Pérez Badia (1997) en el norte de la provincia.

\section{Dichondra micrantha Urb.}

*ALICANTE: San Vicente del Raspeig, Campus universitario, 30SYH1751, $100 \mathrm{~m}, 21-\mathrm{V}$ 1998, M.B. Crespo (ABH 38591).

Planta de origen asiático oriental, cuya utilización en jardinería para céspedes hace que en ocasiones puedan encontrarse ejemplares asilvestrados en terrenos umbrosos o con cierta humedad edáfica. Esta primera localidad alicantina concreta justifica la referencia reciente de su presencia en dicha provincia (Mateo \& Crespo, 1998). Su comportamiento es similar al indicado por Ríos et al. (1992), para las poblaciones de Murcia, y por Carretero \& Aguilella (1995), para las de Valencia. Actualmente se cultiva como césped en numerosos jardines de la ciudad de Alicante.

\section{Galinsoga parviflora Cav.}

*ALICANTE: San Vicente del Raspeig, Campus universitario, 30SYH1752, 85 m, 9-X-1997, M.B. Crespo \& E. Camuñas (ABH 39430).

Especie de origen sudamericano de la que no se tienen referencias previas en la provincia de Alicante. Los ejemplares detectados crecen como adventicios en terrenos húmedos ajardinados, participando en formaciones fragmentarias de Digitario-Setarienion viridis (Sissingh ex Westhoff, Dijk \& Passchier 1946) Oberdorfer 1957

Guizotia abyssinica (L. f.) Cass.

ALICANTE: Alicante, Castillo de Santa Bárbara, 30SYH2047, 90 m, 21-XII-1996, E. Camuñas \& M.B. Crespo (ABH 32254). $2^{\text {a }}$ cita provincial.

Planta africano oriental de la que sólo se conoce una mención previa para las comarcas alicantinas septentrionales (Soler et al., 1995b). Con la nueva localidad se amplía considerablemente su presencia hacia el sur de Alicante. Se presenta de modo asilvestrado a partir de las semillas que se utilizan para alimentar pequeñas aves enjauladas, participando en comunidades de Hordeion leporini. Dado lo escaso y puntual de los ejemplares 
encontrados, deberá considerarse de momento como planta adventicia, lo que coincide con el comportamiento del resto de sus poblaciones valencianas, como ha señalado recientemente Guara (1996).

\section{Lepidium sativum L.}

ALICANTE: Alicante, Castillo de Santa Bárbara, 30SYH2047, 90 m, 21-XII-1996, E. Camuñas \& M.B. Crespo (ABH 32224). $2^{\text {a }}$ cita provincial.

Planta originaria de Oriente Medio, que resulta extremadamente rara en las comarcas alicantinas. Se presenta de modo asilvestrado, probablemente a partir de las semillas que se utilizan para alimentar pequeñas aves domésticas, y participa en comunidades de Hordeion leporini. Dado lo escaso y puntual de los ejemplares encontrados, deberá considerarse de momento como planta adventicia. Recientemente, De la Torre \& Vicedo (1998) recogen su presencia en una localidad cercana a la aquí indicada.

\section{Nothoscordum gracile (Aiton) Stearn [ $=$. fragans}

(Vent.) Kunth]

ALICANTE: Alicante, casco urbano, 30SYH1946, 4 m, 10-V-1998, E. Camuñas \& M.B. Crespo (ABH 39440). Elche, Arenales del Sol, 30SYH13, 29-IV-1991, M.A. Martín (ABH 1573). Guardamar del Segura, 30SYH01, 20-4-1993, I. Pacheco (ABH 11701). Ampliación de área.

Taxón de origen capense que en ocasiones se ha cultivado como planta ornamental y que se encuentra ampliamente extendida por las zonas más cálidas y litorales de la Comunidad Valenciana. Su presencia en Alicante es conocida únicamente en las comarcas septentrionales (Ballester \& Stübing, 1990; Alonso, 1996; Pérez Badia, 1997), donde aparece naturalizada en diversos herbazales de matiz nitrófilo. En las nuevas localidades, que amplían su distribución hasta las áreas más meridionales de la provincia, participa en herbazales de Chenopodion muralis $\mathrm{Br}$--Bl. in Br.-Bl. \& cols. 1936, instalados sobre suelos con notable humedad.

\section{Panicum miliaceum $\mathrm{L}$.}

ALICANTE: Alicante, Barranco de las Ovejas, 30SYH1550, 70 m, 4-VIII-1997, E. Camuñas \& M.B. Crespo (ABH 36717); Alicante, Playa de San Juan, Hotel Sidi, 30SYH2649, 4-IX-1997, E. Camuñas \& M.B. Crespo (ABH 36384). Alicante,
Los Ángeles cerca de Rabasa, 30SYH1849, 75 m, 23-XI-1997, E. Camuñas \& M.B. Crespo (ABH 38551). San Vicente del Raspeig, Los Girasoles, 30SYH1854, 105 m, 21-VIII-1997, E. Camuñas \& A. Juan (ABH 36589). Ampliación de área.

Originario de Asia central y oriental, se presenta a través individuos asilvestrados en herbazales subnitrófilos, pertenecientes a diversas comunidades de Ruderali-Secalietea $\mathrm{Br}$ - $\mathrm{Bl}$. in $\mathrm{Br}$-B1. et al. 1936. Esta planta había sido citada previamente por Rigual (1984), quien la indicó como "escapada de cultivo" en los alrededores de la ciudad de Alicante. Más recientemente, Pérez Badia (1997) la menciona como subespontánea en las comarcas septentrionales de la provincia. Parece, por tanto, que esta planta no encuentra facilidades para su naturalización, dado que su presencia siempre se debe a individuos aislados que no forman poblaciones notables.

Paspalum saurae (Parodi) Parodi

*ALICANTE. Alicante, La Albufereta, 30SYH2450, 15 m, 7-IX-1997, E. Camuñas \& M.B. Crespo (ABH 38384). San Vicente del Raspeig, Campus universitario, 30SYH1752, 100 m, 22-XI1994, M.B. Crespo (ABH 13921).

Planta sudamericana cuya presencia en la Península Ibérica fue dada a conocer por Carretero (1987) de zonas ajardinadas de Valencia. En las nuevas localidades alicantinas se ha localizado en céspedes instalados sobre suelos con alta humedad, donde forma poblaciones muy densas. Sin embargo, no se ha visto que sus poblaciones ganen terreno con rapidez, por lo que no es previsible su naturalización a corto plazo.

\section{Pennisetum villosum $\mathrm{R}$. Br.}

*ALICANTE: Alicante, Castillo de Santa Bárbara (muralla), 30SYH2047, 100 m, 11-I-1997, E. Camuñas \& M.B. Crespo (ABH 32186); ibidem, 13-V-1992, G. Sirvent (ABH 4373).

Planta originaria, al parecer, de Etiopía, de la cual existe una referencia previa (Carretero \& Esteras, 1983), correspondiente a ejemplares introducidos para fijar los taludes de la autopista A7 a su paso por Valencia. La población de Alicante se encuentra integrada en el AristidoHyparrhenietum pubescentis Rivas Mart. et Alcaraz in Alcaraz 1984, y según observaciones directas no 
se ha expandido desde su localización en 1992. Ello se debe, presumiblemente, a que esta especie requiere unos niveles hídricos considerables, que no encuentra con facilidad en los territorios alicantinos.

\section{Physalis peruviana $\mathrm{L}$.}

*ALICANTE: Alicante, Bco. de las Ovejas, 30SYH1550, 60 m, 21-IV-1998, E. Camuñas \& M.B. Crespo (ABH 38550).

Curioso neófito de origen sudamericano, cuya presencia en la flora de la provincia de Valencia era conocida desde antiguo a partir de la referencia dada por Borja (1951) como arvense en la Casella de Alcira. En la nueva localidad alicantina se presenta formando parte de herbazales higronitrófilos de Silybion mariani Rivas Mart. in Rivas Mart. et al. 1992, instalados en el cauce de un barranco con curso de agua más o menos continuo. Aunque esta planta se ha cultivado para el aprovechamiento de sus frutos (Bolòs \& Vigo, 1996), no tenemos constancia de dicho uso en Alicante.

\section{Sesamum indicum $\mathrm{L}$.}

*ALICANTE: Alicante, Barranco de las Ovejas, 30SYH1550, 70 m, 21-IX-1996, M.B. Crespo \& E. Camuñas (ABH 30064).

Planta de origen incierto, seguramente paleotropical, de la que no se tiene constancia explícita de su presencia en la Península Ibérica, a pesar de que Tutin (1972) la indica genéricamente en este territorio. Los ejemplares encontrados viven en una zona con gran humedad y alta nitrificación, en comunidades de Plantaginetalia majoris Tüxen \& Preising in Tüxen 1950. No se tiene noticia sobre el cultivo de esta planta actualmente en Alicante.

\section{Setaria gracilis Kunth}

ALICANTE: Alicante, Camino de la Costa, La Albufereta, 30SYH2350, 15 m, 7-IX-1997, E. Camuñas \& M.B. Crespo (ABH 36460). $2^{\text {a }}$ cita provincial.

Taxón de origen norteamericano cuya primera referencia se sitúa en el norte de la provincia de Alicante, concretamente en el marjal de Pego-Oliva (Soler et al., 1995a). Con la nueva localidad indicada se amplía considerablemente su distribución hacia el sur de la provincia, donde participa en herbazales higro-nitrófilos de Plantaginetalia majoris, instalados en bordes de caminos sometidos a inundación temporal.

\section{Tropaeolum majus L.}

ALICANTE: Alicante, río Monnegre, 30SYH2060, 130 m, 5-V-1997, E. Camuñas \& J.C. Cristóbal (ABH 34927).

Planta procedente de Sudamérica de la que existen muy pocas localidades concretas en la provincia de Alicante (Vicedo \& De la Torre, 1997) y que corresponden siempre a ejemplares herborizados en las proximidades a habitaciones humanas. En la nueva localidad aportada se presenta asilvestrada en cañaverales de Convolvuletalia sepium Tüxen 1950, instalados sobre suelos inundables.

Verbesina encelioides (Cav.) Benth. \& Hook. f. ALICANTE: Elche, 30SYH0341, $150 \mathrm{~m}, 7$ VIII-1991, M.B. Crespo \& M.D. Lledó (ABH 3887). Guardamar, hacia Los Montesinos, 30S Y H0315, 22 m, 11-XII-1996, M.B. Crespo et al. (ABH 32557). $2^{\mathrm{a}}$ cita provincial.

Planta norteamericana cuya presencia se conoce desde hace años en la desembocadura del río Segura cerca de Guardamar (Bolòs \& Sierra, 1981). Con las localidades aquí aportadas se amplía hacia el norte y hacia el interior su distribución alicantina. Se presenta formando parte de comunidades subnitrófilas viarias, instaladas sobre suelos ligeramente salinos, en compañía de Piptatherum miliaceum (L.) Coss., Limonium parvibracteatum Pignatti, Salsola genistoides Juss. ex Poir. y Suaeda vera Forssk., entre otros. Según todos los datos esta planta parece extenderse lentamente por el litoral alicantino.

Wedelia glauca (Ortega) O. Hoffm. ex Hicken

* AliCANTE: Alicante, Babel, prox. Gasolinera ctra. Elche, 30SYH1846, 5 m, 13-XI1997, E. Camuñas et al. (ABH 38553).

Especie sudamericana, cuyas primeras referencias ibéricas fueron dadas por Carretero (1988) para la provincia de Valencia. La nueva población vive en un terreno improductivo, en una zona de encharcamiento temporal, donde forma un manto continuo prácticamente uniespecífico, sólo acompañada por Polygonum equisetiforme Sibth. \& Sm., Aster squamatus (Spreng.) Hieron. y Atriplex prostrata Boucher ex DC. 


\section{BIBLIOGRAFÍA}

ALONSO, M.A. -1996- Flora y vegetación del Valle de Villena (Alicante). Inst. Cult. Gil-Albert. Alicante.

BALLESTER, G. y G. STÜBING - 1990- Sierra del Carrascal de Alcoy. Flora y vegetación. Cuadernos de la Naturaleza 1. Alicante.

BOLÒS, O. y E. SIERRA -1981- Verbesina encelioides al Migjorn Valencià. Butll. Inst. Catalana Hist. Nat., Sec. Bot. 46: 157-159.

BOLÒS, O. y J. VIGO -1996- Flora dels Països Catalans 3. Barcino. Barcelona.

BORJA, J. -1951- Estudio fitográfico de la Sierra de Corbera (Valencia). Anales Inst. Bot. Cavanilles 9: 361-483.

BRUMMITT, R.K. \& C.E. POWELL-1992-Authors of Plant Names. Royal Botanic Gardens, Kew.

CALDUCH, M. -1973- Nota sobre Bidens aurea (Aiton) Sherff. Lagascalia 3(1): 59-60.

CARRETERO, J.L. - 1987- Paspalum saurae (Parodi) Parodi, una gramínea nueva para Europa. Anales Jard. Bot. Madrid 44: 175-176.

CARRETERO, J.L. - 1988- Wedelia glauca (Ortega) O. Hoffm. ex Hicken en España. Anales Jard. Bot. Madrid 45: 346-347.

CARRETERO, J.L. y A. AGUILELLA - 1995- Flora y vegetación nitrófilas del término municipal de la ciudad de Valencia. Publ. Excmo. Ayuntamiento de Valencia.

CARRETERO, J.L. y F.J. ESTERAS - 1983- Algunas gramíneas de interés corológico para la provincia de Valencia. Collect. Bot. (Barcelona) 14: 215 219.

CASTROVIEJo, S., M. LAÍNZ, G. LÓPEZ, P. MONTSERRAT, F. MUÑOZ, J. PAIVA Y L. VILLAR -1986-98- Flora iberica. vols. 1-8. Real Jardín Botánico, CSIC. Madrid.

DE LA TORRE, A. y M.A. VICEDO -1998Fragmenta chorologica occidentalia, 6621-6624. Anales Jard. Bot. Madrid 56(1): 143.

GUARA, M. -1996- Guizotia abyssinica (L. fil.) Cass. en Valencia. ¿Naturalizada o adventicia? Fl. Montiber. 4: 81-82.

MATEO, G. y M.B. CRESPO -1998- Manual para la determinación de la flora valenciana. Monogr. Fl. Montiber. 3. Valencia.

MATEO, G. y M.B. CRESPO -1988- Fragmenta chorologica occidentalia, 1745-1759. Anales Jard. Bot. Madrid 45(1): 332-333.
NEBOT, J.R., A. DE LA TORRE, G., MATEO y F. ALCARAZ -1990- Materiales para la actualización del catálogo florístico de la provincia de Alicante. Anales Biol. (Murcia) 16: 99-129.

PÉREZ BADIA, R., A. DE LA TORRE, L. SERRA y M.B.CRESPO -1994- Notas corológicas sobre plantas alicantinas. Fontqueria 40: 25-29.

PÉREZ BADIA, R. -1997-Flora vascular y vegetación de la comarca de la Marina Alta. Publ. Inst. Cult. Gil-Albert. Alicante

RIGUAL, A. -1972- Flora y vegetación de la provincia de Alicante. Inst. Estud. Alicantinos. Alicante.

RIGUAL, A. -1984- Flora y vegetación de la provincia de Alicante. $2^{\mathrm{a}}$ ed. Inst. Cult. GilAlbert. Alicante.

RÍOS, S., A. ROBLEDO y F. ALCARAZ -1992Notas sobre la flora alóctona del sureste ibérico, I (España). Anales Biol. (Murcia) 18: 95-102.

SOLER, J.X., L. SERRA, G. MATEO y M.B. CRESPO - 1995a- Adiciones a la flora alicantina. Fl. Montiber. 1: 23-28.

SOLER, J.X., B. PÉREZ ROCHER, G. MATEO y L. SERRA - 1995b- Fragmenta chorologica occidentalia, 5479-5509. Anales Jard. Bot. Madrid 53(1): 113-114.

TUTIN, T.G. -1972- Sesamum L. Pp. 284. In: Flora Europaea, 3. T.G. Tutin et al. Eds. Cambridge University Press. Cambridge.

VICEDO, M.A. y A. DE LA TORRE -1997- La Sierra de Crevillente: flora y vegetación. Publ. Inst. Cult. Gil-Albert. Alicante.

Aceptado para su publicación en Octubre de 1998

Dirección de los autores: Departamento de Ciencias Ambientales y Recursos Naturales (Botánica). Universidad de Alicante. Apdo. 99. E-03080 Alicante. E-mail: crespo@carn.ua.es 